\title{
Depth-Dependent Glycosaminoglycan Concentration in Articular Cartilage by Quantitative Contrast-Enhanced Micro-Computed Tomography
}

\author{
Daniel Mittelstaedt' ${ }^{\prime}$ and $Y$ ang $X i a^{\prime}$
}

\begin{abstract}
Objective. A quantitative contrast-enhanced micro-computed tomography (qCECT) method was developed to investigate the depth dependency and heterogeneity of the glycosaminoglycan (GAG) concentration of ex vivo cartilage equilibrated with an anionic radiographic contrast agent, Hexabrix. Design. Full-thickness fresh native ( $n=19$ in 3 subgroups) and trypsindegraded $(n=6)$ articular cartilage blocks were imaged using micro-computed tomography $(\mu C T)$ at high resolution $(I 3.4$ $\mu \mathrm{m}^{3}$ ) before and after equilibration with various Hexabrix bathing concentrations. The GAG concentration was calculated depth-dependently based on Gibbs-Donnan equilibrium theory. Analysis of variance with Tukey's post hoc was used to test for statistical significance $(P<0.05)$ for effect of Hexabrix bathing concentration, and for differences in bulk and zonal GAG concentrations individually and compared between native and trypsin-degraded cartilage. Results. The bulk GAG concentration was calculated to be $74.44 \pm 6.09$ and $11.99 \pm 4.24 \mathrm{mg} / \mathrm{mL}$ for native and degraded cartilage, respectively. A statistical difference was demonstrated for bulk and zonal GAG between native and degraded cartilage $(P<0.032)$. A statistical difference was not demonstrated for bulk GAG when comparing Hexabrix bathing concentrations $(P>0.3214)$ for neither native nor degraded cartilage. Depth-dependent GAG analysis of native cartilage revealed a statistical difference only in the radial zone between $30 \%$ and $50 \%$ Hexabrix bathing concentrations. Conclusions. This nondestructive qCECT methodology calculated the depth-dependent GAG concentration for both native and trypsin-degraded cartilage at high spatial resolution. qCECT allows for more detailed understanding of the topography and depth dependency, which could help diagnose health, degradation, and repair of native and contrived cartilage.
\end{abstract}

\section{Keywords}

micro-computed tomography $(\mu \mathrm{CT})$, contrast agent (Hexabrix [loxaglate]), glycosaminoglycans (GAG), cartilage, osteoarthritis (OA)

\section{Introduction}

Articular cartilage is comprised of an extracellular matrix (ECM) primarily composed of water, collagen fibers, and proteoglycans (PG) composed of negatively charged glycosaminoglycans $(\mathrm{GAG}){ }^{1,2}$ The interaction between these macromolecules creates a hydraulic permeability allowing diffusion from the synovial fluid to obtain nutrients essential to maintaining integrity of the tissue..$^{3-5}$ The collagen fibers are oriented depth-dependently into 3 subtissue zones with respect to the surface defined as: parallel (superficial zone [SZ]), random (transitional zone [TZ]), and perpendicular (radial zone [RZ]). The collagen fibers in the ECM are intertwined with PG molecules depth-dependently in different concentrations. ${ }^{6}$ PG macromolecules are composed of a protein core composed of highly electronegative side chains, chondroitin and keratan sulfate, and hyaluronan, which binds aggrecan monomers to form highly charged aggregates that help regulate the diffusion of solutes attributed to the hydrophilic fixed charge density (FCD) and contributes to pore size with a link protein. ${ }^{7-9}$ Reduced GAG concentration, either natural or contrived, exhibits both an increased diffusion rate and increased equilibrium concentration of an anion due to the reduced FCD. ${ }^{10-15}$ Reduction of GAG alters the collagen fiber structural integrity and has been associated with the initial degradation and/or onset of diseases (e.g., osteoarthritis [OA]) and quantitative depth-dependent

'Department of Physics and Center for Biomedical Research, Oakland University, Rochester, MI, USA

\section{Corresponding Author:}

Yang Xia, Ph.D., Department of Physics, Oakland University, 276 Hannah Hall, Oakland Rochester, MI 48309, USA.

Email: xia@oakland.edu 
measurements may aid in diagnosing and treating nonhealthy cartilage. . $^{12,16,17}$

Electroneutrality of cartilage in a bathing solution of ionic, anionic, or neutral molecules of different molecular sizes has been investigated to determine the GAG concentration using the Gibbs-Donnan theory. ${ }^{7,18,19}$ Microscopic magnetic resonance imaging ( $\mu \mathrm{MRI}$ ) has been shown to measure the depth-dependent FCD of cartilage attributed to the GAG concentration, including delayed gadoliniumenhanced MRI of cartilage (dGEMRIC), glycosaminoglycans chemical exchange saturation transfer, diffusion tensor imaging, and sodium $\left({ }^{23} \mathrm{Na}\right)$ imaging. ${ }^{20-24}$ The dGEMRIC method calculates GAG based on cartilage in equilibrium with a negatively charged paramagnetic contrast agent gadolinium diethylene triamine pentaacetic acid $\left(\operatorname{Gd}(D T P A){ }^{2-}\right){ }^{20,25} \mathrm{~T} 1$ measurements before and after equilibration with Gd can be used to calculate the GAG depthdependently based on Gibbs-Donnan equilibrium theory. The negatively charged Gd ions will diffuse depth-dependently inversely related to the GAG in order to reach electroneutrality attributed to the repulsion of the negative charges.

Recently, micro-computed tomography $(\mu \mathrm{CT})$ has been used to assess the integrity and predict changes in GAG within healthy and degraded (e.g., biochemically and biomechanically) articular cartilage using radiographic contrast agents of various molecular sizes and charge. ${ }^{10,14,26-36}$ Palmer et $a l .{ }^{26}$ investigated the use of Hexabrix (Malinckrodt, St. Louis, MO) equilibration in cartilage that could qualitatively predict differences in GAG content based on changes in x-ray attenuation developing a technique called equilibrium partitioning of an ionic contrast agent via $\mu \mathrm{CT}$. Recently, differences in x-ray attenuations were found for different aged and enzymatic degradation of cartilage using $\mu \mathrm{CT} .^{31,37}$ Trypsin was used to enzymatically degrade the cartilage, which has been shown to remove $\sim 90 \%$ of the GAG and has some disruption to the collagen fibril network. ${ }^{13,38}$ Kokkonen et al. ${ }^{10}$ investigated the time-dependent diffusion of contrast agents and observed a difference in x-ray attenuation and diffusion flux for Hexabrix in healthy and mechanically damaged cartilage.

The aim of the present study was to quantify and compare of the depth and zonal dependencies of the GAG concentration of native and degraded ex vivo articular cartilage using quantitative contrast enhanced micro-computed tomography (qCECT) at high resolution.

\section{Materials and Methods}

\section{Cartilage and Hexabrix Preparation}

Fresh humeral heads were harvested, with the approval of the local regulatory committee, within 5 hours of sacrifice from three healthy and mature ( $\sim 1-2$ years old) canines that were used in an unrelated scientific study. The load bearing region of the humerus was divided into 3-mm sections using a diamond saw (MTI Corporation, Richmond, CA) and then sectioned into full-thickness rectangular blocks $\sim 3$ $\times 2 \times 5 \mathrm{~mm}$, which were immersed in $154 \mathrm{mM}$ physiological saline containing $1 \%$ protease inhibitor $(\mathrm{S}+\mathrm{PI})$ overnight until experimentation (for a maximum of 4 days, from the harvesting). Nineteen blocks were designated for no treatment (native) and an additional 6 blocks were selected for trypsin degradation (degraded). Trypsin (Sigma-Aldrich, St. Louis, MO) solutions were prepared by adding $1.23 \mathrm{mg}$ of trypsin per $1 \mathrm{~mL}$ of S + PI. Full thickness cartilage-bone specimens were immersed in trypsin for approximately 6 hours and then placed overnight in an S + PI bath to remove any excess trypsin.

The cartilage contrast agent solution was prepared by adding Hexabrix (Ioxaglate, Mallinckrodt Inc., St Louis, $\mathrm{MO}$ ) to S + PI in serial concentrations for use in the phantoms and bathing solutions. Hexabrix contains $320 \mathrm{mg} / \mathrm{mL}$ of bound iodine and is an ionic dimer that is composed of 2 salts, namely, $393 \mathrm{mg} / \mathrm{mL}$ of ioxaglate meglumine and 196 $\mathrm{mg} / \mathrm{mL}$ of ioxaglate sodium, which both dissociate into -1 charged ioxaglate with a molecular weight of $1269 \mathrm{~g} / \mathrm{mol}$. In order to determine the relationship between the x-ray attenuation and concentration of Hexabrix, Hexabrix phantoms $(0 \%-100 \%$ in $10 \%$ increments including $5 \%$ ) were imaged to obtain a calibration curve for the x-ray attenuation.

\section{Micro-Computed Tomography Experiments}

All $\mu \mathrm{CT}$ experiments were performed using Skyscan1174 (Bruker, Kontich, Belgium) with identical experimental parameters: $40 \mathrm{kV}, 110 \mathrm{mAs}, 5$ averages, $0.3^{\circ}$ rotation step, $180^{\circ}$ rotation, $0.2 \mathrm{~mm} \mathrm{Al}$ filter, and $652 \times 512$ data matrix, which took approximately 30 minutes to acquire approximately 608 images with a $13.4 \mu \mathrm{m}$ isotropic voxel size. All scans were reconstructed using NRecon (Bruker, Kontich, Belgium) with the same parameters and a global intensity threshold was kept constant for all scans to include the full range of attenuation values. Figure 1A shows a representative 3-dimensional (3D) image of a native cartilage-bone specimen equilibrated in Hexabrix.

Two-dimensional (2D) grayscale images were converted to Hounsfield units (HU) based on the property that air = $-1000 \mathrm{HU}$ and water $=0 \mathrm{HU} .{ }^{39}$ The conversion of linear attenuation to $\mathrm{HU}$ was based on the calibration of air and water:

$$
\mathrm{HU}=30.84 \times(\text { Gray value })-1000
$$

Each cartilage block was gently blotted before the experiments to remove any excess S + PI or Hexabrix and secured in a radiopaque custom-made airtight holder containing an S + PI soaked gauze to prevent evaporation 


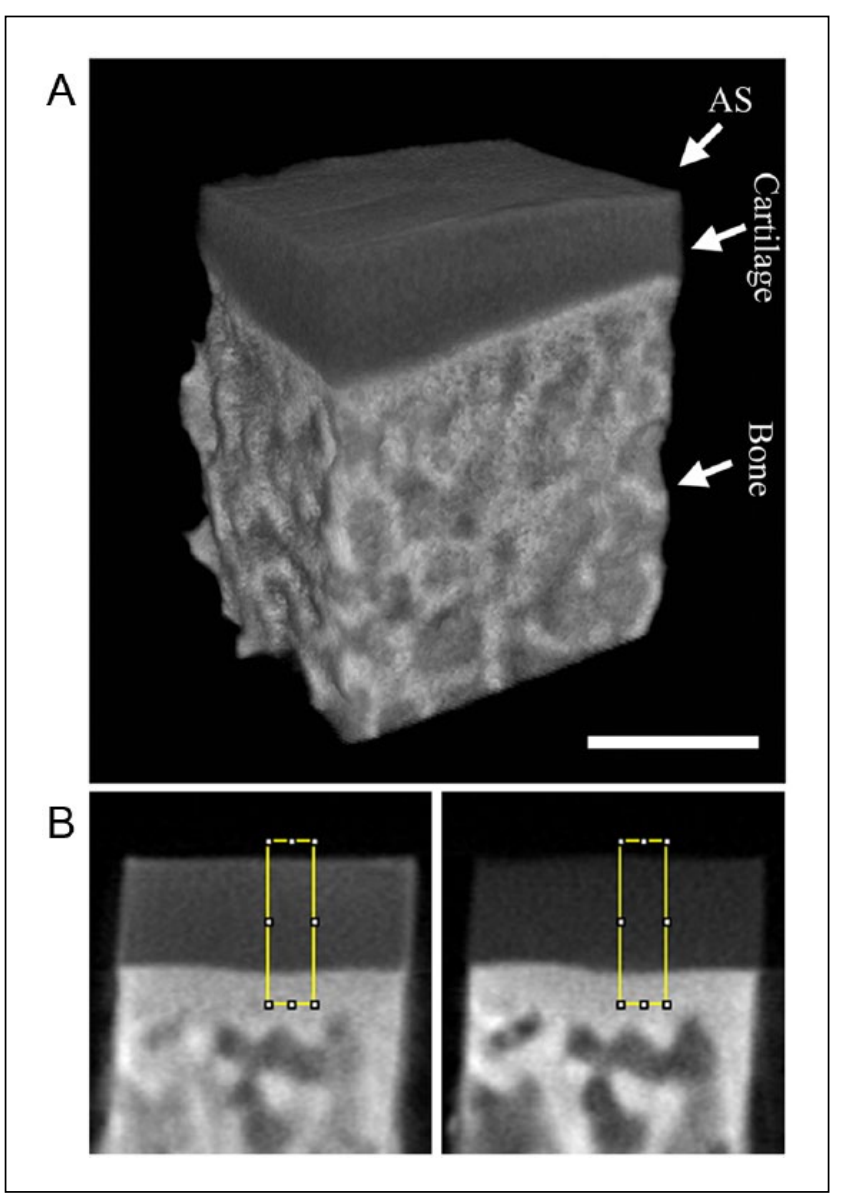

Figure I. (A) A 3-dimensional representation of native cartilage equilibrated in $40 \%$ Hexabrix bathing solution. (B) A representation of the 2-dimensional sagittal image registration of the identical native cartilage block imaged after equilibration $40 \%$ Hexabrix (left) and "baseline," without Hexabrix (right), including a 20-pixel wide region of interest. The scale bar represents approximately I mm for (A) and (B). AS, articular surface.

during the experimentation. The cartilage blocks were manually aligned so that the articular surface was parallel to the direction of $\mathrm{x}$-rays to minimize surface beam hardening. Each block was scanned after being immersed in $\mathrm{S}+$ PI to obtain a "baseline" scan (i.e., no Hexabrix), then immersed overnight ( $\sim 24$ hours) in a known Hexabrix bath concentration until equilibration then imaged again to measure the increase in x-ray attenuation. Preliminary investigations of eight native cartilage blocks were used to investigate Hexabrix bathing concentrations from $10 \%$ to $100 \%$ and found that $30 \%, 40 \%$, and $50 \%$ obeyed the Gibbs-Donnan theory and took approximately 24 hours to reach equilibrium. ${ }^{14,29}$ Subsequently, 19 fresh native blocks were chosen to investigate Hexabrix solutions $30 \%(n=5)$, $40 \%(n=9)$, and 50\% $(n=5)$ and 2 additional blocks per Hexabrix group were selected to investigate the effect of trypsin degradation.

\section{Image and Data Analysis}

Cartilage and phantom images were analyzed using ImageJ (National Institutes of Health, Bethesda, MD) and KaleidaGraph (Synergy, Reading, PA). ${ }^{40}$ Ten consecutive sagittal images near the central region of each phantom and cartilage block were averaged to improve the signal to noise ratio. A $20 \times 20$ pixel region of interest (ROI) was chosen for the phantoms to find the average attenuation for each Hexabrix concentration group. Figure 1A shows a representative 3D image of a cartilage block after equilibration in a $40 \%$ Hexabrix bathing solution. The image registration for the cartilage images before and after Hexabrix equilibration was determined manually by aligning both images in 3D space then finding similar landmark characteristics in the 2D sagittal images. A 20-column ROI, including the full-thickness of cartilage, was used to calculate the average depth-dependent attenuation at similar locations for each cartilage block before and after equilibration with Hexabrix as shown in Figure 1B. The average attenuation was calculated (mean \pm standard deviation) at each depth to obtain a depth-dependent profile from the articular surface to the tissue-bone interface. The bulk GAG was defined as the full-thickness average. Zonal thicknesses were based on percentages depth-wise from the surface and the RZ is divided equally into 2 halves: $10 \%$ of cartilage is SZ, $10 \%$ is $\mathrm{TZ}, 40 \%$ is RZ1, and $40 \%$ is RZ2. ${ }^{41}$

The Hexabrix equilibrated cartilage HU profile was subtracted from the baseline $\mathrm{HU}$ profile to obtain the $\Delta \mathrm{HU}$ depth-dependent profile. This value was then converted into the diffused concentrations of Hexabrix based on the calibration of the Hexabrix phantoms. The FCD and GAG depth-dependent profiles were calculated based on the Gibbs-Donnan equilibrium theory ${ }^{20,25}$ :

$$
\begin{gathered}
\mathrm{FCD}=\left[\mathrm{Na}^{+}\right]_{\mathrm{b}} \times\left(\sqrt{\frac{[I]_{\mathrm{t}}}{[I]_{\mathrm{b}}}}-\sqrt{\frac{[\mathrm{I}]_{\mathrm{b}}}{[\mathrm{I}]_{\mathrm{t}}}}\right) \\
{[\mathrm{GAG}]_{\mathrm{t}}=\mathrm{FCD} \times \frac{-502.5}{2}}
\end{gathered}
$$

where FCD is the fixed charge density, $\left[\mathrm{Na}^{+}\right]_{\mathrm{b}}$ is the sodium ion concentration in the bathing solution; $[\mathrm{I}]_{\mathrm{t}}$ and $[\mathrm{I}]_{\mathrm{b}}$ are the Ioxaglate (Hexabrix) concentrations in the tissue and the bath, respectively, and [GAG] is the concentration of GAG in the tissue. The values "502.5" and " 2 " are constants attributed to $2 \mathrm{~mol}$ of negative charges for each mol of disaccharide with molecular weight of $502.5 \mathrm{~g} / \mathrm{mol}$.

\section{Statistical Model}

Using KaleidaGraph and JMP (SAS, Cary, NC), analysis of variance with Tukey's post hoc test was used to determine (1) any effect of the Hexabrix bathing concentration on bulk GAG for native and/or degraded cartilage, (2) any effect on 


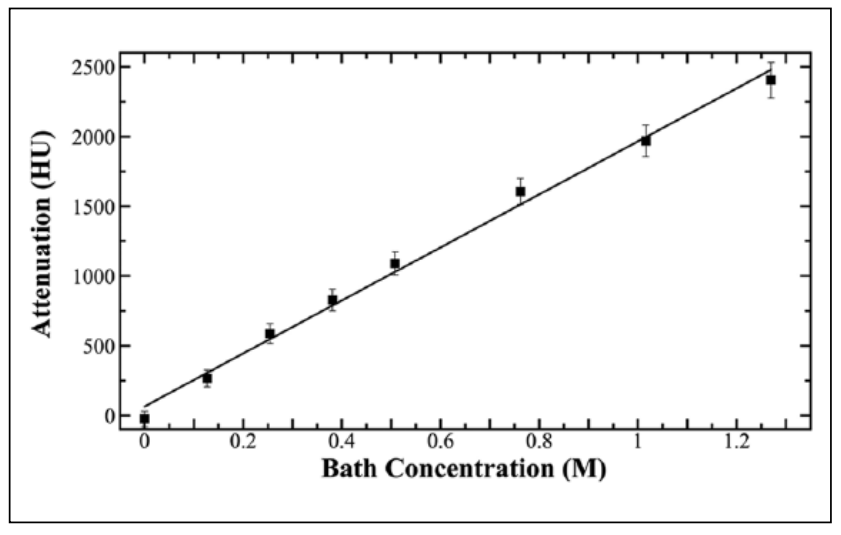

Figure 2. Average x-ray attenuation measurements are plotted from the various Hexabrix concentrations of the phantoms.

bulk GAG from trypsin degradation compared with native cartilage, and (3) differences in zonal depth-dependent GAG concentrations for native and degraded cartilage. Significance was defined as $P<0.05$, and any values $\sim 0.05$ showed a possibility for significance and indicated variation.

\section{Results}

In order to measure the concentration of Hexabrix based on $\mathrm{x}$-ray attenuation from the $\mu \mathrm{CT}$ parameters, a calibration curve was determined by the average attenuation value from the Hexabrix phantoms and plotted in Figure 2. A positive linear relationship was found between the x-ray attenuation and Hexabrix concentrations up to $50 \%$ ( $R=0.997)$ :

$$
y=65.15+1900 x
$$

At higher concentrations, the x-ray attenuations of Hexabrix phantoms were no longer linear (data not shown). ${ }^{14}$ This slope of the linear calibration was used in the cartilage imaging experiments to convert $\mathrm{x}$-ray attenuation to Hexabrix concentration, and used to calculate the $[\mathrm{I}]_{\mathrm{t}}$ in Equation (2) from the depth-dependent $\Delta \mathrm{HU}$ profiles.

Two-dimensional representative sagittal images of native and degraded cartilage immersed in $30 \%, 40 \%$, and $50 \%$ Hexabrix bathing solutions are shown in Figure 3. Morphologically, the lack of intensity variation across the width of all specimens indicates similar diffusion of the contrast agent at each specific depth of the tissue. In addition, small edge enhancement at the boundary of the tissue blocks was due to the beam-hardening effect and/or excess Hexabrix remaining after blotting. Intensity-wise, the degraded samples clearly show a marked increase in x-ray

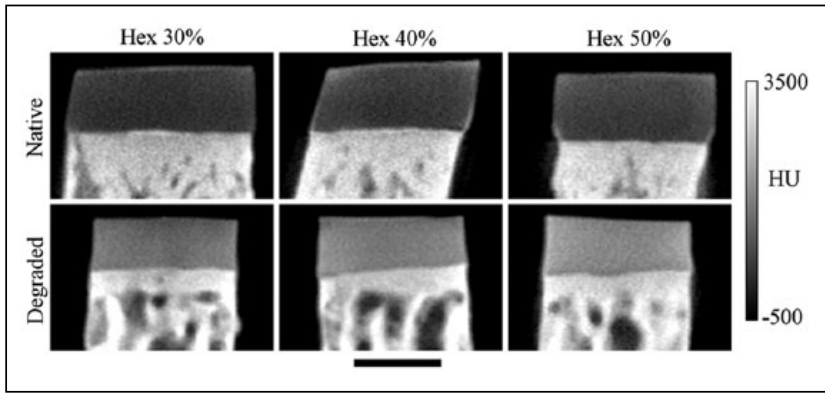

Figure 3. A representative set of 2-dimensional sagittal images of cartilage equilibrated in $30 \%, 40 \%$, and $50 \%$ Hexabrix bathing solutions for native and trypsin-degraded samples.

attenuation compared with the native cartilage regardless of bath concentration. The native cartilage was measured up to $500 \mu \mathrm{m}$ and the degraded was measured up to $450 \mu \mathrm{m}$ including the full-thickness, and the discrepancy is explained later in the Discussion section.

The depth-dependent profiles of cartilage equilibrated with Hexabrix (filled symbols) and "baseline" (open symbols) are plotted in Figure 4 for native and degraded cartilage. The "baseline" profiles do not show a marked difference either among Hexabrix bathing concentrations or between native and degraded cartilage. The depth-dependent HU profiles in both native and degraded cartilage (Fig. 4A and B) show increased attenuation for increasing Hexabrix bathing solutions (e.g., $30 \%<40 \%<50 \%$ ). This increase in attenuation is consistent with cartilage having a larger influx of Hexabrix ions from an increased bathing concentration for native and degraded cartilage and is shown in Figure 4C and D, respectively.

Using Equations (2) to (4), the HU profiles were converted into the $\left[\mathrm{I}_{\mathrm{t}}\right.$ and $\mathrm{FCD}$ profiles (Fig. 4C-F), which represented the depth-dependent diffusion of Hexabrix (Ioxaglate) into the tissue ([I $]_{\mathrm{t}}$ ) at equilibrium and the $\mathrm{FCD}$ in the tissue. In native cartilage, the depth-dependent Hexabrix concentration of negative ions (Fig. 4C) shows an inverse relation to the FCD (Fig. 4E). In contrast, there was an increase in the depth-dependent Hexabrix concentration in degraded cartilage (Fig. 4D) that was related to the lower depth-dependent FCD (Fig. 4F). Finally, Figure 4G and $\mathbf{H}$ shows the GAG concentration when the cartilage was equilibrated in $30 \%, 40 \%$, and $50 \%$ Hexabrix of healthy and degraded cartilage, respectively. The native cartilage shows a positive linear trend in the depth-dependent GAG measurements, and the degraded cartilage shows almost complete removal of GAG. The average bulk GAG for the native cartilage is $74.44 \pm 6.09 \mathrm{mg} / \mathrm{mL}$ and for the trypsin-degraded samples is $11.99 \pm 4.24 \mathrm{mg} / \mathrm{mL}$. Wang et al., ${ }^{42}$ from our lab, used some of the adjacent humeri blocks to measure the GAG content using a T1 dGEMRIC protocol with various concentrations of Gd and found the bulk GAG to be $76.6 \pm$ 


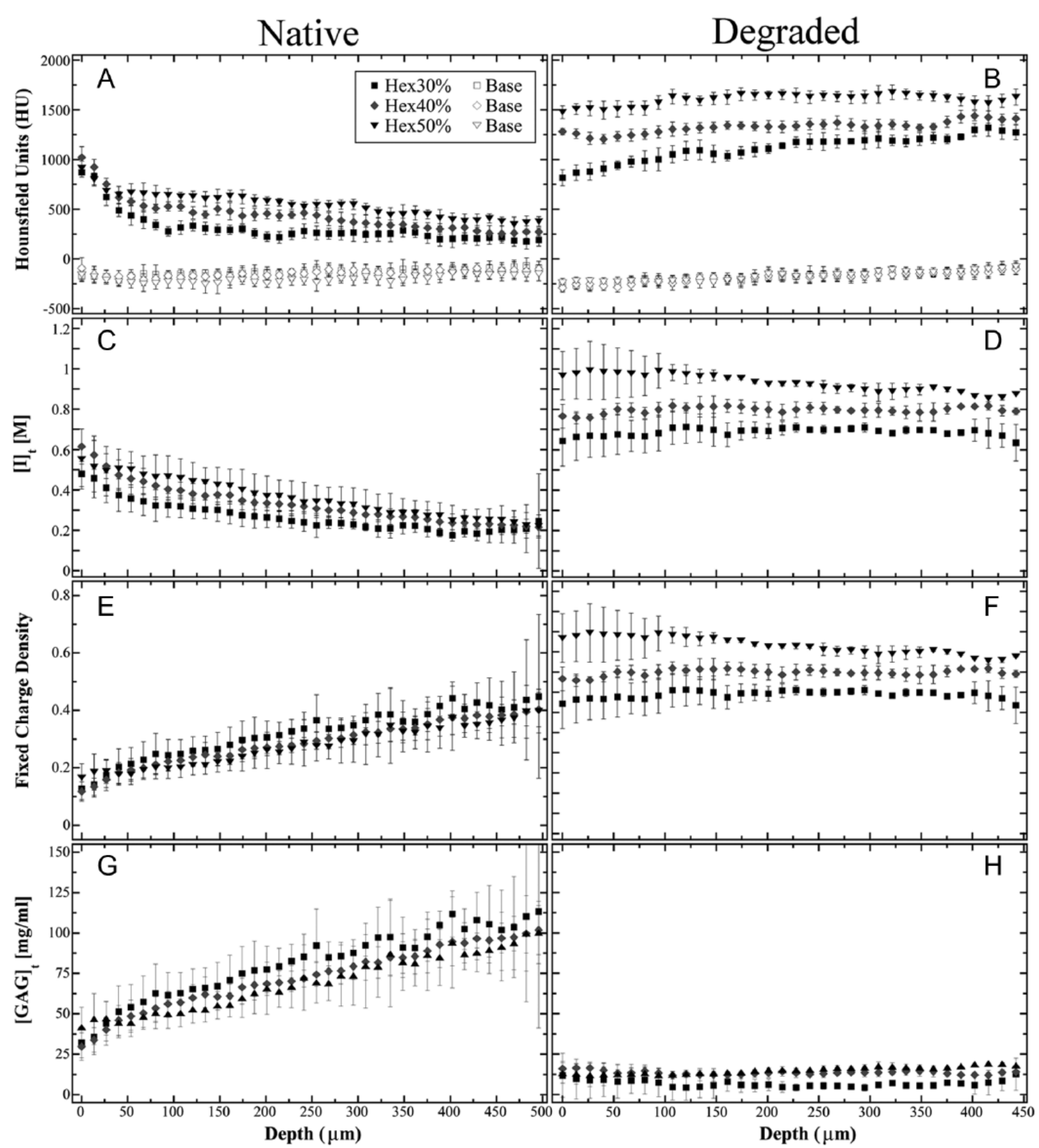

Figure 4. The depth-dependent cartilage profiles after equilibration with Hexabrix (30\%, 40\%, and 50\%) and the same "baseline" (open symbol) profile for native $(\mathbf{A}, \mathbf{C}, \mathbf{E}, \mathbf{G})$ and trypsin-degraded $(\mathbf{B}, \mathbf{D}, \mathbf{F}, \mathbf{H})$ samples. The HU profiles $(\mathbf{A}, \mathbf{B})$ are from the microcomputed tomography experiments. The diffusion of Hexabrix $(\mathbf{C}, \mathbf{D})$ is calculated from the calibration curve. The fixed charge density $(\mathbf{E}, \mathbf{F})$ and glycosaminoglycan $(\mathbf{G}, \mathbf{H})$ profiles are calculated based on quantitative contrast-enhanced micro-computed tomography.

3.3 and $16.2 \pm 5.9 \mathrm{mg} / \mathrm{mL}$ for native and trypsin-degraded cartilage, respectively, and matched well with the GAG measurements from the inductively coupled plasma optical emission spectrometer (ICP-OES). The comparison between ex vivo experiments using $\mu \mathrm{CT}$ and $\mu \mathrm{MRI}$ were consistent and measured similar depth-dependent profiles of the GAG content of articular cartilage (data not shown).

Statistical analyses ( $P$ values) for the zonal GAG comparisons are shown in Table 1 and there was a demonstration of statistical significance for the comparisons. The 
Table I. ANOVA $P$ Values for Bulk and Zonal GAG Comparisons. ${ }^{a}$

\begin{tabular}{|c|c|c|c|c|c|c|c|c|c|c|}
\hline & \multicolumn{3}{|c|}{ (a) Native vs. Native } & \multicolumn{3}{|c|}{ (b) Degraded vs. Degraded } & \multicolumn{4}{|c|}{ (c) Native vs. Degraded } \\
\hline & & Hex $40 \%$ & Hex $50 \%$ & & Hex $40 \%$ & Hex $50 \%$ & & Hex30\% & Hex $40 \%$ & Hex $50 \%$ \\
\hline \multirow[t]{3}{*}{ Bulk } & Hex30\% & 0.5189 & 0.3214 & Hex $30 \%$ & 0.9684 & 0.9225 & $\mathrm{Hex} 30 \%$ & $<0.0001$ & $<0.0001$ & $<0.0001$ \\
\hline & Hex $40 \%$ & & 0.9897 & Hex $40 \%$ & & 1 & Hex $40 \%$ & $<0.0001$ & $<0.0001$ & $<0.0001$ \\
\hline & & & & & & & Hex $50 \%$ & $<0.0001$ & $<0.0001$ & $<0.0001$ \\
\hline \multirow[t]{3}{*}{ SZ } & Hex30\% & 0.999 & $\mathrm{I}$ & Hex30\% & 0.977 & 0.999 & Hex $30 \%$ & 0.005 & 0.005 & 0.005 \\
\hline & Hex $40 \%$ & & 0.997 & Hex $40 \%$ & & 0.999 & Hex $40 \%$ & 0.028 & 0.032 & 0.028 \\
\hline & & & & & & & Hex $50 \%$ & 0.011 & 0.012 & 0.011 \\
\hline \multirow[t]{3}{*}{ TZ } & Hex30\% & 0.826 & 0.554 & Нex30\% & 0.994 & 0.998 & Hex30\% & $<0.0001$ & 0.0004 & 0.003 \\
\hline & Hex $40 \%$ & & 0.976 & Hex $40 \%$ & & I & Hex $40 \%$ & 0.0005 & 0.0016 & 0.0107 \\
\hline & & & & & & & Hex $50 \%$ & 0.0004 & 0.0012 & 0.0085 \\
\hline \multirow[t]{3}{*}{ RZI } & Hex30\% & 0.3791 & 0.03 I 8 & Hex30\% & 0.9285 & 0.844 & Hex30\% & 0.0001 & 0.0001 & 0.0001 \\
\hline & Hex $40 \%$ & & 0.4408 & Hex $40 \%$ & & 0.9999 & Hex $40 \%$ & $<0.0001$ & $<0.0001$ & $<0.0001$ \\
\hline & & & & & & & Hex $50 \%$ & $<0.0001$ & $<0.0001$ & $<0.0001$ \\
\hline \multirow[t]{3}{*}{ RZ2 } & Hex30\% & 0.8496 & 0.0515 & Нex30\% & 0.961 & 0.7475 & Hex30\% & $<0.0001$ & $<0.0001$ & $<0.0001$ \\
\hline & Hex $40 \%$ & & 0.2077 & Hex $40 \%$ & & 0.9931 & Hex $40 \%$ & $<0.0001$ & $<0.0001$ & $<0.0001$ \\
\hline & & & & & & & Hex $50 \%$ & $<0.0001$ & $<0.0001$ & $<0.0001$ \\
\hline
\end{tabular}

ANOVA = analysis of variance; GAG = glycosaminoglycan; Hex = Hexabrix; RZ = radial zone; $\mathrm{SZ}=$ superficial zone; $\mathrm{TZ}=$ transitional zone.

${ }^{a}$ The values in boldface highlight the variation found in native cartilage between Hexabrix bathing concentrations of $30 \%$ and $50 \%$.

native cartilage only demonstrated a statistical difference or variation when comparing GAG concentrations of $30 \%$ and $50 \%$ in RZ1 $(P=0.0318)$ and RZ2 $(P=0.0515)$ shown in Table 1a. The $P$ value of 0.0515 is relatively close to the limit of $P<0.05$ and could show variation among the samples. GAG concentrations of degraded samples showed no statistical difference $(P>0.7475)$ between various Hexabrix bathing concentrations shown in Table 1b. Comparisons between native and degraded GAG concentrations demonstrated statistical differences $(P<0.032)$ for bulk, every zone, and every Hexabrix bath concentration shown in Table 1c.

\section{Discussion}

In this study, we investigated the possibility to quantify the bulk and depth-dependent GAG concentrations from fresh (never frozen) ex vivo native and degraded cartilage using qCECT. $^{43,44}$ The full-thickness bulk GAG of native cartilage was calculated to be $74.44 \pm 6.09 \mathrm{mg} / \mathrm{mL}$ and for the trypsin-degraded samples the GAG was $11.99 \pm 4.24 \mathrm{mg} /$ $\mathrm{mL}$, which are found to be consistent with the investigations in the literature and previous $\mu \mathrm{MRI}$ studies. ${ }^{19,20,25}$ There was no statistical difference in bulk GAG arising from the difference in Hexabrix bathing concentration, agreeing well with Silvast et $a .^{27}$ A statistical difference of GAG concentrations was demonstrated in the RZ of fresh native humeral cartilage when comparing Hexabrix bathing concentrations of $30 \%$ and $50 \%$. The GAG difference in RZ1 could allow for a better understanding of the depthdependent variations of healthy cartilage that may lead to better detection of degradation. The ability to detect depthdependent changes in GAG concentration noninvasively can only aid in the understanding of cartilage health, degradation, growth, and repair., $4,5,15,30,34,41,45$

\section{Hexabrix Phantoms}

In order to relate Hexabrix diffusion in the tissue with attenuation, preliminary experiments were performed to optimize experimental parameters for Hexabrix attenuation in phantoms and native full-thickness cartilage. The slope calculated from the average Hexabrix phantom attenuation averages was shown in Equation (4) having a correlation of 0.997 demonstrating high accuracy and precision similar to calibrations found by Yoo et al. ${ }^{14}$ who used both charged (Ioxaglate) and uncharged (Iopromide) contrast agents. Kallioniemi et al. ${ }^{36}$ also found similar relationships between $\mathrm{x}$-ray attenuation and contrast agent concentration using a contrast agentenhanced peripheral quantitative computed tomography. These similar calibrations from both clinical and $\mu \mathrm{CT}$ resolutions allows for improved correlations and verifications using various contrast agents. These HU calibrations of Hexabrix concentration to attenuation values depend on equipment protocol, experimental settings, and reconstruction settings. This calibration is similar to the calibration of the $R$-value (i.e., diffusivity) used to calculate the GAG concentration by the dGEMRIC method. Although there are different physical processes for the calibration of qCECT and dGEMRIC, the ability to have multidisciplinary quantitative correlations allows for increased verification and reproducibility of the GAG concentration in articular cartilage. ${ }^{17,20,46}$ 


\section{Bulk GAG Measurements Using qCECT}

qCECT was used to quantitatively measure the x-ray attenuation and to calculate the GAG concentration in native and degraded articular cartilage in equilibration with a negatively charged contrast agent (Hexabrix). The equilibration was found to follow Gibbs-Donnan equilibrium theory and the bulk GAG concentration was calculated to be $74.44 \pm$ 6.09 and $11.99 \pm 4.24 \mathrm{mg} / \mathrm{mL}$ for native and degraded cartilage, respectively. This measured difference is attributed to a decreased GAG concentration attributed to the degradation from trypsin. Yoo et al. ${ }^{14}$ used a clinical CT scanner and investigated quantification of GAG in cartilage and found a marked decrease in GAG due to Ioxaglate, charged ion, but no difference due to Iopromide, uncharged ion, from trypsin degradation. These GAG measurements are similar to various other techniques for measuring native and degraded articular cartilage including dGEMRIC, ${ }^{23} \mathrm{Na}$, dimethylmethylene blue assays, quantitative proteomics, and ICP-OES. ${ }^{6,14,19,24,42}$

\section{Depth Dependency}

A clearer understanding of the depth dependency of articular cartilage is crucial in determining health and the alterations that occur from degradation, disease, or injury (e.g., $\mathrm{OA}$, aging, sports injuries, etc.) and monitoring the efficacy of repair and regrowth. ${ }^{4,30,47}$ The statistical difference demonstrated between native and degraded cartilage allows for the possibility of qCECT to detect depth-dependent changes from damage (e.g., from aging) or disease (e.g., OA). The depth-dependent diffusion and equilibration of various Hexabrix bathing concentrations shows the effects that are attributed to the FCD, pore size, collagen cross-linking, and steric interactions..$^{10,26,48}$ Kokkonen et al ${ }^{10}$ investigated the diffusion flux and equilibration and found that the main reason for the diffusion of Ioxaglate and iodide was attributed to the FCD and investigated the effects of cross-linking and steric interactions. They showed that the FCD has a greater effect on the equilibration than the cross-links; however, the collagen cross-linking is important to cartilage integrity. ${ }^{49}$

In this current study, the deep tissue subzones RZ1 and RZ2 demonstrated a statistical difference in GAG concentration of fresh native articular cartilage when comparing $30 \%$ and $50 \%$ Hexabrix bathing concentrations. Since there was no other effect from Hexabrix concentration in the zonal GAG measurements, the difference in the RZ could be attributed to the variation of the tissue properties. Müller et $a l .{ }^{6}$ investigated the depth-dependent protein concentrations of human tibial cartilage using mass spectroscopy and found aggrecan and link protein had a linear increasing concentration with depth and other depth-dependent proteins that could affect diffusion in different zones. The linear increase of aggrecan is consistent with the increasing FCD found in this study. Although qCECT cannot distinguish individual types of proteins, the interactions between Hexabrix and various proteins may be the reason for the diffusion difference in the $\mathrm{RZ}$ of fresh native cartilage. The detection of these subtle changes attributed to tissue integrity allow for future investigations into the detection and clearer understanding of tissue health and degradation.

\section{Micro-Computed Tomography Comparisons}

The ability to quantify the depth-dependent GAG concentration will allow $\mu \mathrm{CT}$ to have quantitative comparisons with other techniques. Silvast et al. ${ }^{27}$ investigated the diffusion and partition function of Ioxaglate and iodide in cartilage plugs from the patella and found no difference in attenuation among bathing concentrations. The investigation of the partition function is important for in vivo cartilage experiments since equilibration is not required and the contrast agent diffusion rate is related to the GAG concentration. Bansal et al. ${ }^{33}$ investigated the electrostatic attraction of 2 cationic (CA1+ and $\mathrm{CA} 4+)$ contrast agents and electrostatic repulsion of 1 anionic $(\mathrm{CC} 2)$ contrast agent and found that the cations provided a more accurate prediction of the GAG concentration. The ability to use positive, neutral, and negative charged particles allows for a better understanding of the diffusion and equilibration in cartilage related to the overall tissue properties. The use of positively charged contrast agents could be used to investigate the variation of GAG we found in the RZ of fresh native cartilage because of the limited diffusion in the deep zones of cartilage. $^{50}$

\section{Cartilage Thickness}

The fresh native cartilage was measured up to $500 \mu \mathrm{m}$, which includes the full-thickness, and the degraded tissue was measured up to $450 \mu \mathrm{m}$. Trypsin can cause cartilage to shrink after degradation, and the "baseline" and Hexabrix cartilage images did not show much observable difference in full-thickness measurements as well as initial thickness measurements before each scan. Torzilli et al. ${ }^{38}$ reported that the hydrophilic PG molecules are restrained from expanding by the tensile strength of the collagen fiber network. This restraint as well as the removal of the hydrophilic PG could be possible causation of the $50 \mu \mathrm{m}$ differences of the full-thickness observed in this study.

\section{Experimental Limitations}

There were several limitations of this study. This study used a relatively low sample size; however, the statistical analyses demonstrated high significance, and comparable results were similar to the other studies in the literature. The requirements for Gibbs-Donnan equilibrium were not 
satisfied when there was a large mismatch in osmolarities between cartilage and bathing solution and were not further investigated. There may have been a slight beam hardening effect because no algorithm was used for mathematical correction; however, the sample was aligned to minimize the beam hardening effect on the articular surface. Also, the trypsin-degraded samples should have an increased beam hardening effect, which was not observed. This quantitative imaging method using $\mu \mathrm{CT}$ is currently limited to ex vivo samples, because of the requirement of Hexabrix equilibration that may not possible to obtain for in vivo cartilage. This study used only one form of degradation and one negatively charged radiographic contrast agent. However, to the best of our knowledge, this is the first $\mu \mathrm{CT}$ study to quantitatively measure the depth-dependent GAG concentration using Gibbs-Donnan equilibrium and investigations within the subtissue zones of articular cartilage. Finally, we did not carry out the histological assay for GAG determination in this project. However, the specimens used in this project came from the same tissue source (the same age animals of the same species) for our laboratory for more than 12 years. We have used these specimens in various imaging and nonimaging projects, many quantitative comparisons among multidisciplinary techniques. For example, Wang et al. ${ }^{42}$ compared microscopic MRI and ICP-OES quantification using the nearly identical tissue. The measurement from $\mu \mathrm{CT}$ in this project was in excellent agreement with all of our previous quantitative measurements.

\section{Conclusions}

In conclusion, CT has recently become an invaluable tool for monitoring the integrity for both in vivo and ex vivo cartilage. ${ }^{15,16,33,37}$ qCECT was used in this project for the first time to quantify the depth-dependent GAG concentration and investigate the zonal variations from both healthy and degraded cartilage. The ability to quantify the depthdependent concentration of GAG molecules in cartilage will allow possibilities for a better understanding and detection of disease and degradation. High-resolution (e.g., 13.4 $\mu^{3}$ ) studies are helpful in detecting differences in subtissue zones and have the possibility of being applied to lower resolution (e.g., $0.200 \mathrm{~mm}^{3}$ ) images to help diagnose topological differences in cartilage health, degradation, and repair. ${ }^{51}$ The ability to quantitatively compare cartilage integrity using qCECT could allow for better comparisons of multidisciplinary investigations, which may allow for a more detailed understanding of in vivo, ex vivo, and in situ cartilage.

\section{Acknowledgments and Funding}

The authors thank the National Institutes of Health (NIH) for the support from a R01 grant. The authors are grateful to Dr. Hani Sabbah (Henry Ford Hospital, Detroit, MI) for providing the specimens. The authors would like to thank Dr. Clifford Les (Michigan State University) for statistical expertise, Dr. George Artzberger (Graham Health Center, Oakland University) for help in obtaining the contrast agent, and Dr. Nian Wang (Oakland University) for help in applying the Gibbs-Donnan theory. Also, the authors greatly appreciate Mr. David Kahn, Dr. Ji Hyun Lee, and Mr. Farid Badar for editing and critical discussions. This project is made possible by a R01 research grant (AR052353; PI: Y Xia) from the NIH.

\section{Declaration of Conflicting Interests}

The author(s) declared no potential conflicts of interest with respect to the authorship and/or publication of this article.

\section{Ethical Approval}

This study was approved by our local institutional review board, IACUC.

\section{References}

1. Ratcliffe A, Mow V. Articular cartilage. In: Comper WD, editor. Extracellular matrix. Amsterdam, Netherlands: Harwood Academic Publishers; 1996. p. 235-302.

2. Maroudas A, Venn M. Chemical composition and swelling of normal and osteoarthrotic femoral head cartilage. II. Swelling. Ann Rheum Dis. 1977;36:399-406.

3. O'Hara B, Urban J, Maroudas A. Influence of cyclic loading on the nutrition of articular cartilage. Ann Rheum Dis. 1990;49:536-9.

4. Brittberg M, Winalski CS. Evaluation of cartilage injuries and repair. J Bone Joint Surg Am. 2003;85(Suppl 2):58-69.

5. Buckwalter J, Mankin H. Articular cartilage repair and transplantation. Arthritis Rheum. 1998;41:1331-42.

6. Müller C, Khabut A, Dudhia J, Reinholt FP, Aspberg A, Heinegård $\mathrm{D}$, et al. Quantitative proteomics at different depths in human articular cartilage reveals unique patterns of protein distribution. Matrix Biol. 2014;40:34-45.

7. Lesperance LM, Gray ML, Burstein D. Determination of fixed charge density in cartilage using nuclear magnetic resonance. J Orthop Res. 1992;10:1-13.

8. Maroudas A. Distribution and diffusion of solutes in articular cartilage. Biophys J. 1970;10:365-79.

9. Maroudas A, Muir H, Wingham J. The correlation of fixed negative charge with glycosaminoglycan content of human articular cartilage. Biochim Biophys Acta. 1969;177:492-500.

10. Kokkonen H, Jurvelin J, Tiitu V, Töyräs J. Detection of mechanical injury of articular cartilage using contrast enhanced computed tomography. Osteoarthritis Cartilage. 2011;19:295-301.

11. Xia Y, Farquhar T, Burton-Wurster N, Vernier-Singer M, Lust G, Jelinski L. Self-diffusion monitors degraded cartilage. Arch Biochem Biophys. 1995;323:323-8.

12. Venn M, Maroudas A. Chemical composition and swelling of normal and osteoarthrotic femoral head cartilage. I. Chemical composition. Ann Rheum Dis. 1977;36:121-9.

13. Bartholomew JS, Handley CJ, Lowther DA. The effects of trypsin treatment on proteoglycan biosynthesis by bovine articular cartilage. Biochem J. 1985;227:429-37. 
14. Yoo HJ, Hong SH, Choi J-Y, Lee IJ, Kim SJ, Choi J-A, et al. Contrast-enhanced CT of articular cartilage: experimental study for quantification of glycosaminoglycan content in articular cartilage. Radiology. 2011;261:805-12.

15. Silvast TS, Jurvelin JS, Lammi MJ, Toyras J. pQCT study on diffusion and equilibrium distribution of iodinated anionic contrast agent in human articular cartilage - associations to matrix composition and integrity. Osteoarthritis Cartilage. 2009; 17:26-32.

16. Boerckel JD, Mason DE, McDermott AM, Alsberg E. Microcomputed tomography: approaches and applications in bioengineering. Stem Cell Res Ther. 2014;5:144.

17. Batiste DL, Kirkley A, Laverty S, Thain LM, Spouge AR, Holdsworth DW. Ex vivo characterization of articular cartilage and bone lesions in a rabbit ACL transection model of osteoarthritis using MRI and micro-CT. Osteoarthritis Cartilage. 2004;12:986-96.

18. Huttunen J, Kokkonen H, Jurvelin J, Töyräs J, Kaipio J. Estimation of fixed charge density and diffusivity profiles in cartilage using contrast enhanced computer tomography. Int J Numer Method Biomed Eng. 2014;98:371-90.

19. Xia Y, Zheng S, Bidthanapally A. Depth-dependent profiles of glycosaminoglycans in articular cartilage by $\mu \mathrm{MRI}$ and histochemistry. J Magn Reson Imaging. 2008;28:151-7.

20. Zheng S, Xia Y. The impact of the relaxivity definition on the quantitative measurement of glycosaminoglycans in cartilage by the MRI dGEMRIC method. Magn Reson Med. 2010;63:25-32.

21. Ling W, Regatte RR, Navon G, Jerschow A. Assessment of glycosaminoglycan concentration in vivo by chemical exchange-dependent saturation transfer (gagCEST). Proc Natl Acad Sci U S A. 2008;105:2266-70.

22. Deng X, Farley M, Nieminen MT, Gray M, Burstein D. Diffusion tensor imaging of native and degenerated human articular cartilage. Top Magn Reson Imaging. 2007;25: 168-71.

23. Gillis A, Gray M, Burstein D. Relaxivity and diffusion of gadolinium agents in cartilage. Magn Reson Med. 2002;48: 1068-71.

24. Shapiro EM, Borthakur A, Gougoutas A, Reddy R. ${ }^{23} \mathrm{Na}$ MRI accurately measures fixed charge density in articular cartilage. Magn Reson Med. 2002;47:284-91.

25. Bashir A, Gray M, Hartke J, Burstein D. Nondestructive imaging of human cartilage glycosaminoglycan concentration by MRI. Magn Reson Med. 1999;41:857-65.

26. Palmer AW, Guldberg RE, Levenston ME. Analysis of cartilage matrix fixed charge density and three-dimensional morphology via contrast-enhanced microcomputed tomography. Proc Natl Acad Sci U S A. 2006;103:19255-60.

27. Silvast TS, Jurvelin JS, Tiitu V, Quinn TM, Töyräs J. Bath concentration of anionic contrast agents does not affect their diffusion and distribution in articular cartilage in vitro. Cartilage. 2013;4:42-51.

28. Xie L, Lin AS, Levenston ME, Guldberg RE. Quantitative assessment of articular cartilage morphology via EPIC- $\mu \mathrm{CT}$. Osteoarthritis Cartilage. 2009;17:313-20.

29. Bansal P, Joshi N, Entezari V, Grinstaff M, Snyder B. Contrast enhanced computed tomography can predict the glycosaminoglycan content and biomechanical properties of articular cartilage. Osteoarthritis Cartilage. 2010;18: 184-91.

30. Siebelt M, van Tiel J, Waarsing J, Piscaer T, van Straten M, Booij R, et al. Clinically applied CT arthrography to measure the sulphated glycosaminoglycan content of cartilage. Osteoarthritis Cartilage. 2011;19:1183-9.

31. Xie L, Lin AS, Guldberg RE, Levenston ME. Nondestructive assessment of sGAG content and distribution in normal and degraded rat articular cartilage via EPIC- $\mu \mathrm{CT}$. Osteoarthritis Cartilage. 2010;18:65-72.

32. Stewart RC, Bansal PN, Entezari V, Lusic H, Nazarian RM, Snyder BD, et al. Contrast-enhanced CT with a highaffinity cationic contrast agent for imaging ex vivo bovine, intact ex vivo rabbit, and in vivo rabbit cartilage. Radiology. 2013;266:141-50.

33. Bansal PN, Joshi NS, Entezari V, Malone BC, Stewart $\mathrm{RC}$, Snyder BD, et al. Cationic contrast agents improve quantification of glycosaminoglycan (GAG) content by contrast enhanced CT imaging of cartilage. J Orthop Res. 2011;29:704-9.

34. Hirvasniemi J, Kulmala K, Lammentausta E, Ojala R, Lehenkari P, Kamel A, et al. In vivo comparison of delayed gadolinium-enhanced MRI of cartilage and delayed quantitative CT arthrography in imaging of articular cartilage. Osteoarthritis Cartilage. 2013;21:434-42.

35. Cockman M, Blanton C, Chmielewski P, Dong L, Dufresne T, Hookfin E, et al. Quantitative imaging of proteoglycan in cartilage using a gadolinium probe and microCT. Osteoarthritis Cartilage. 2006;14:210-4.

36. Kallioniemi AS, Jurvelin JS, Nieminen MT, Lammi MJ, Töyräs J. Contrast agent enhanced pQCT of articular cartilage. Phys Med Biol. 2007;52:1209-19.

37. Piscaer T, Waarsing J, Kops N, Pavljasevic P, Verhaar $\mathrm{J}$, van Osch $\mathrm{G}$, et al. In vivo imaging of cartilage degeneration using $\mu \mathrm{CT}$-arthrography. Osteoarthritis Cartilage. 2008;16:1011-7.

38. Torzilli PA, Arduino JM, Gregory JD, Bansal M. Effect of proteoglycan removal on solute mobility in articular cartilage. J Biomech. 1997;30:895-902.

39. Schneider U, Pedroni E, Lomax A. The calibration of CT Hounsfield units for radiotherapy treatment planning. Phys Med Biol. 1996;41:111-124.

40. Schneider CA, Rasband WS, Eliceiri KW. NIH Image to ImageJ: 25 years of image analysis. Nat Methods. 2012;9:671-5.

41. Lee JH, Badar F, Kahn D, Matyas J, Qu X, Chen CT, et al. Topographical variations of the strain-dependent zonal properties of tibial articular cartilage by microscopic MRI. Connect Tissue Res. 2014;55:205-16.

42. Wang N, Chopin E, Xia Y. The effects of mechanical loading and gadolinium concentration on the change of T1 and quantification of glycosaminoglycans in articular cartilage by microscopic MRI. Phys Med Biol. 2013;58:4535-47.

43. Kahn D, Les C, Xia Y. Effects of cryopreservation on the depth-dependent elastic modulus in articular cartilage and implications for osteochondral grafting. J Biomech Eng. 2015;137:054502. 
44. Zheng S, Xia Y, Bidthanapally A, Badar F, Ilsar I, Duvoisin N. Damages to the extracellular matrix in articular cartilage due to cryopreservation by microscopic magnetic resonance imaging and biochemistry. Top Magn Reson Imaging. 2009;27:648-55.

45. Xia Y, Wang N, Lee J, Badar F. Strain-dependent T1 relaxation profiles in articular cartilage by MRI at microscopic resolutions. Magn Reson Med. 2011;65:1733-7.

46. Reece D, Lin A, Guldberg RE. Contrast-enhanced microCT imaging. In: Neu CP, Genin GM, editors. Handbook of imaging in biological mechanics. Boca Raton, FL: CRC Press; 2015. p. 127-42.

47. Surowiec RK, Lucas EP, Ho CP. Quantitative MRI in the evaluation of articular cartilage health: reproducibility and variability with a focus on T2 mapping. Knee Surg Sports Traumatol Arthrosc. 2014;22:1385-95.

48. Diab M, Wu J, Eyre D. Collagen type IX from human cartilage: a structural profile of intermolecular cross-linking sites. Biochem J. 1996;314:327-32.

49. Vickers SM, Squitieri LS, Spector M. Effects of cross-linking type II collagen-GAG scaffolds on chondrogenesis in vitro: dynamic pore reduction promotes cartilage formation. J Tissue Eng. 2006;12:1345-55.

50. Arkill K, Winlove C. Solute transport in the deep and calcified zones of articular cartilage. Osteoarthritis Cartilage. 2008;16:708-14.

51. Xia Y. Resolution 'scaling law' in MRI of articular cartilage. Osteoarthritis Cartilage. 2007;15:363-5. 